\title{
Antiganglioside antibody in patients with Guillain-Barré syndrome who show bulbar palsy as an initial symptom
}

\author{
Michiaki Koga, Nobuhiro Yuki, Koichi Hirata
}

\begin{abstract}
Objectives-To identify valuable antiganglioside antibodies that support the diagnosis of Guillain-Barré syndrome (GBS) and its variants in patients showing bulbar palsy as an initial symptom.

Methods-Medical records of 602 patients with GBS or its variants were reviewed. Fifteen patients had bulbar palsy as an initial symptom. Serum antibodies against GM1, GM1b, GD1a, GalNAcGD1a, GT1a, and GQ1b were examined in 13 of them.

Results-Serum antiganglioside antibodies were positive in $11(85 \%)$ patients. IgG anti-GT1a $(n=8 ; 62 \%)$ and anti-GM1b $(n=7 ; 54 \%)$ antibodies were often present, whereas all the patients had low or no anti-GM1 antibody activity. High antiGD1a and anti-GQ1b IgG antibody titres were also present in some patients, but most had higher IgG antibody titres to GM1b or GT1a. All five patients with high IgG antibody titre to GM1b or GT1a only had had antecedent diarrhoea. Some patients with pharyngeal-cervicalbrachial weakness (PCB) had IgG antibody to GT1a which did not cross react with GQ1b. Other patients with PCB had antibody to GT1a which cross reacted with GQ1b or antibody to GM1b, but antiGM1b and anti-GT1a antibodies were not associated with the presence of bulbar palsy. All the patients who had no IgG antiganglioside antibodies recovered completely.

Conclusions-Measurement of serum IgG anti-GT1a and anti-GM1b antibodies gives helpful support for the diagnosis of GBS and its variants when there is early involvement of the oropharyngeal function independently of other neurological findings which appear as the illness progresses.
\end{abstract}

(F Neurol Neurosurg Psychiatry 1999;66:513-516)

Keywords: Guillain-Barré syndrome; bulbar palsy; antiganglioside antibody

Bulbar palsy may be an initial clinical sign in several neurological or non-neurological disorders. ${ }^{1}$ Dysphagia and dysarthria often appear at the beginning of botulism and diphtheria. Myasthenia gravis, brainstem vascular disturbance, multiple sclerosis, and tumour invasion to the vagus nerve sometimes show oropharyngeal dysfunctions early in the clinical course. Guillain-Barré syndrome (GBS) also may have the initial neurological sign of bulbar palsy, although very rarely. ${ }^{2}$ Patients with GBS should be treated with plasmapheresis or intravenous immunoglobulin as soon as possible to shorten the duration of disability..$^{3-6}$ It is therefore important to differentiate GBS and its variants, which show early oropharyngeal dysfunctions, from other disorders. Physiological studies and CSF testing, however, may detect no abnormalities in some patients with GBS who show bulbar palsy early.?

Measurement of serum antiganglioside antibodies should prove useful for supporting the diagnosis of GBS or its variants. The major gangliosides GM1 and GD $1 \mathrm{a}^{8-11}$ and the minor ones GM1b, GalNAc-GD1a, and GT1 $a^{12-17}$ in bovine brain are target molecules for the autoantibodies found in GBS and its variants. The presence of IgG anti-GM1 antibody, strong supportive evidence for the diagnosis of GBS, however, is not common in patients with GBS with cranial nerve involvement. ${ }^{18}$ Furthermore, there are only a few reports on serum antiganglioside antibodies in patients with GBS or its variants who show bulbar palsy as an initial symptom, ${ }^{17-21}$ and it is not clear which antiganglioside antibodies are useful for differentiating GBS with early oropharyngeal dysfunction from other disorders. To assess the diagnostic value of the antiganglioside antibodies present in patients with GBS and its variants who had bulbar palsy as an initial symptom, we examined the frequencies of detectable serum antibodies to various gangliosides, including minor ones, in these patients and the relation of antiganglioside antibodies to clinical features.

\section{Patients and methods}

PATIENTS

Medical records were reviewed of 387 patients with GBS, 156 with Fisher's syndrome (FS), 36 with Bickerstaff's brainstem encephalitis (BBE), and 23 with acute ophthalmoparesis, all 
Table 1 Clinical features of patients with GBS with the initial symptom of bulbar palsy

\begin{tabular}{|c|c|c|c|c|c|c|c|c|}
\hline No & Age/sex & Antecedent events & Symptoms at onset & $O P$ & $F P$ & Limb weakness & Therapy & Outcome (time after onset) \\
\hline 1 & $33 / \mathrm{M}$ & URTI & $\mathrm{BP}$, weakness in legs & - & + & $+($ arms $=$ legs $)$ & PP & Severe limb weakness ( 10 weeks) \\
\hline 2 & $23 / M$ & Diarrhoea & $\mathrm{BP}$, dysaesthesia & - & + & + (arms $>$ legs $)$ & PP & Unknown \\
\hline 3 & $14 / \mathrm{M}$ & Diarrhoea & $\mathrm{BP}$ & + & + & $+($ arms $=$ legs $)$ & PP, others & Moderate arm weakness, mild OP (4 months) \\
\hline 4 & $34 / \mathrm{F}$ & URTI, diarrhoea & BP, dysaesthesia & + & + & - & $\mathrm{PP}$ & Mild OP ( 5 months) \\
\hline 5 & $37 / \mathrm{M}$ & Diarrhoea & $\mathrm{BP}$, blurred vision & + & - & + (only in arms) & Steroid iv & Mild OP (4 weeks) \\
\hline 6 & $55 / \mathrm{F}$ & URTI, diarrhoea & $\mathrm{BP}$, nausea, ptosis & + & + & $+($ arms $>$ legs $)$ & Steroid im & No deficit ( 3 months) \\
\hline 7 & $19 / M$ & Absent & $\mathrm{BP}, \mathrm{OP}$ & + & - & - & $\mathrm{PP}$ & Mild OP (3 weeks) \\
\hline 8 & $33 / \mathrm{M}$ & URTI & $\mathrm{BP}$, dysaesthesia & + & + & + (only in arms) & $\mathrm{PP}$ & Mild OP (18 months) \\
\hline 9 & $29 / \mathrm{F}$ & Unknown & $\mathrm{BP}$, dysaesthesia & + & + & - & Not done & Mild OP (4 weeks) \\
\hline 10 & $44 / \mathrm{F}$ & URTI & $\mathrm{BP}$ & + & + & $+($ arms $>$ legs $)$ & IVIg & Moderate limb weakness ( 3 months) \\
\hline 11 & $55 / \mathrm{M}$ & URTI, diarrhoea & $\mathrm{BP}$, gait disturbance & - & + & $+($ arms $=$ legs $)$ & PP & No deficit ( 3 months) \\
\hline 12 & $7 / \mathrm{F}$ & Fever & $\mathrm{BP}$ & + & + & $+($ arms $>$ legs $)$ & IVIg & No deficit (12 months) \\
\hline 13 & $54 / \mathrm{F}$ & URTI & BP, dysaesthesia & - & - & $+($ arms $>$ legs $)$ & Not done & No deficit (4 weeks) \\
\hline
\end{tabular}

URTI=upper respiratory tract infection; $\mathrm{BP}=$ bulbar palsy; $\mathrm{OP}=$ ophthalmoparesis; $\mathrm{FP}=$ facial palsy; $\mathrm{PP}=$ plasmapheresis; $\mathrm{IVIg}=$ intravenous immunoglobulin.

of whom had been referred to our neuroimmunological laboratory between June 1994 and March 1998 for tests for serum antiganglioside antibodies. Diagnoses of GBS, FS, and acute ophthalmoparesis were based on established clinical criteria. ${ }^{722}{ }^{23}$ Patients with BBE fulfilled all the following: (1) presence of external ophthalmoplegia and cerebellar ataxia; (2) consciousness disturbance or presence of long tract signs such as pyramidal signs and hemisensory disturbance; (3) recovery from neurological deficits beginning within 4 weeks of onset; and (4) ability to rule out the diagnosis of cerebral vascular disease, brain tumour, Wernicke's encephalopathy, botulism, multiple sclerosis, or herpes simplex virus encephalitis. Our review of current illness and neurological signs on the day of admission indicated that 15 patients (mean age 34; nine males; six females) had bulbar palsy as an initial symptom (11 patients with GBS, two with FS, one with BBE, and one with acute ophthalmoparesis). Of these 15 patients, serum samples were taken within 4 weeks of the onset of neurological symptoms from $13(87 \%)$ (mean age 34; seven males; six females, table 1 ), who therefore were included in the present study. The details of patients 5 and 6 have been reported elsewhere. ${ }^{17}{ }^{21}$ To monitor the functional prognosis of the patients, follow up faxes were sent when possible to the physicians who treated the patients during the recovery phase of the illness. Because serum antibodies against GM1b were frequent in patients with GBS with early involvement of the lower cranial nerves, we also examined 175 consecutive patients with GBS $(n=124)$ or FS $(n=51)$ to determine whether the presence of bulbar palsy is significantly related to serum anti-GM1b IgG and $\operatorname{IgM}$ antibodies.

ENZYME LINKED IMMUNOSORBENT ASSAY

We measured the serum antibodies to GM1, GM1b, GD1a, GalNAc-GD1a, GT1a, and GQ1b using the enzyme linked immunosorbent assay (ELISA) described elsewhere. ${ }^{17}$ Serum was considered positive when the antibody titre was $\geqslant 500$

ABSORPTION STUDY

This study was done as described elsewhere ${ }^{17}$ with minor modifications. Antiganglioside antibodies were absorbed in microtitre wells coated with 10 pmole portions of ganglioside. Absorption rates were expressed as percentages of the optical densities obtained with and without absorption treatment.

\section{Results}

CLINICAL FEATURES (TABLE 1)

Eleven $(92 \%)$ of 12 patients for whom clinical data were available had had antecedent symptoms, of which upper respiratory infection was the most frequent $(n=7 ; 58 \%)$ and diarrhoea the second $(n=6 ; 50 \%)$. The patients often complained of dysaesthesia in the distal extremities at the time bulbar palsy appeared. Facial palsy and ophthalmoplegia were frequent (facial palsy, 77\%; ophthalmoparesis, $69 \%$ ). Muscle weakness was present in some or all limbs in $10(77 \%)$ patients, of whom seven (54\%) had arm dominant weakness but no leg dominant weakness. Only one (patient 5), as reported elsewhere, ${ }^{17}$ met the clinical criteria for pharyngeal-cervical-brachial weakness (PCB) proposed by Ropper. ${ }^{2}$ The other six (patients 2, 6, 8, 10, 12, and 13) with arm dominant weakness also had severe weakness for neck flexion, but did not fulfill the criteria for PCB because areflexia and/or muscle weakness were present throughout the lower limbs. Babinski's sign, drowsiness, ophthalmoparesis, and ataxia were present temporarily in patient 8 , in whom the diagnosis of BBE was made. Assisted ventilation was required in five patients $(2,3,8,11$, and 12$)$. Four patients $(6$, 11,12 , and 13) made complete recoveries within 3 to 12 months after onset, whereas moderate or severe limb weakness remained in patients 1,3 , and 1010 weeks to 4 months after onset. Bulbar palsy disappeared in all the patients for whom clinical prognosis data were available. Mild ophthalmoparesis was present in six patients $(3,4,5,7,8$, and 9) from 3 weeks to 18 months after onset, but they had no problems in carrying out daily activities.

ANTIGANGLIOSIDE ANTIBODIES

Serum antiganglioside antibodies were positive in $11(85 \%)$ of 13 patients (table 2$)$. IgG antiGT1a ( $=8 ; 62 \%)$ and anti-GM1b ( $=7 ; 54 \%)$ antibodies were frequent, whereas anti-GM1 antibody activity was low or not detected. High anti-GD1a and anti-GQ1b IgG antibody titres were found in some patients, but all except one (patient 10) had higher IgG antibody titres to GM1b or GT1a.

Patients were classified into four groups (groups A-D) according to their IgG antiganglioside antibodies (table 2). Group A (patients 
Table 2 Antiganglioside antibody titres in GBS patients with the initial symptom of bulbar palsy

\begin{tabular}{|c|c|c|c|c|c|c|c|c|c|c|c|c|}
\hline \multirow[b]{2}{*}{ No } & \multicolumn{6}{|c|}{ IgG Antibodies to: } & \multicolumn{6}{|c|}{ IgM Antibodies to: } \\
\hline & $G M 1$ & $G M 1 b$ & $G D 1 a$ & GalNAc-GD1a & GT1a & $G Q 1 b$ & $G M 1$ & $G M 1 b$ & GD1a & GalNAc-GD1a & GT1a & $G Q 1 b$ \\
\hline 1 & 500 & 256000 & 64000 & - & - & - & 500 & 500 & - & 500 & 500 & - \\
\hline 2 & 500 & 128000 & 1000 & - & - & - & - & - & - & 500 & - & - \\
\hline 3 & - & 128000 & - & 500 & 4000 & 500 & 1000 & 32000 & 500 & 32000 & - & 500 \\
\hline 4 & - & 4000 & - & - & 500 & - & - & 1000 & - & - & 500 & - \\
\hline 5 & - & - & - & - & 32000 & - & - & - & - & - & - & - \\
\hline 6 & - & 1000 & - & - & 32000 & 500 & - & - & - & - & - & - \\
\hline 7 & - & - & - & - & 500 & - & - & - & - & - & - & - \\
\hline 8 & - & 4000 & 64000 & - & 1024000 & 256000 & - & - & - & - & - & - \\
\hline 9 & - & 500 & - & - & 32000 & 8000 & - & 1000 & - & 500 & 500 & 500 \\
\hline 10 & - & - & - & - & 2000 & 8000 & - & - & - & - & - & 500 \\
\hline 11 & - & - & - & - & - & - & - & - & - & - & - & - \\
\hline 12 & - & - & - & - & - & - & - & - & - & - & - & - \\
\hline 13 & - & - & - & - & - & - & - & - & - & - & 1000 & 500 \\
\hline
\end{tabular}

Only titres of $\geqslant 500$ are shown.

1-4) had a marked increase in IgG anti-GM1b antibody, which was not absorbed by GT1a or GQ1b, but in some patients were absorbed by GD1a or GM1 (table 3). In group B (patients 5-7), IgG anti-GT1a antibodies that did not cross react with GQ1b were detected as described elsewhere. ${ }^{1721}$ Group C (patients 8-10) had IgG anti-GT1a antibodies which cross reacted with GQ1b and sometimes with GD1a (table 3). Group D (patients 11-13) had no $\mathrm{IgG}$ antibodies to the gangliosides used in this study. Thin layer chromatography (TLC) with immunostaining was performed using serum samples from two patients ( 1 and 8 ). Serum IgG from patient 1 reacted with GM1b ganglioside but not with others such as GM1, GD1a, GT1a, or GQ1b on TLC (data not shown). The IgG from patient 8 reacted strongly with GT1a and more weakly with GQ1b (data not shown).

Three $(75 \%)$ of the four patients in group A had had antecedent diarrhoea, and all showed facial palsy. Two had moderate to severe residual muscle weakness 10 to 16 weeks after onset, and one made a complete recovery, except for mild ophthalmoparesis, 5 months after onset. In groups B and C, ophthalmoparesis and arm dominant limb weakness were often present, and minimal residual symptoms of ophthalmoparesis remained. In Group D, ophthalmoparesis was less common and all three patients made a complete recovery. Five $(71 \%)$ of the seven (patients 2, 5, 6, 8, 10,12, and 13) with PCB-like symptoms had high IgG antiganglioside antibody titre; two had IgG anti-GT1a antibodies which did not cross react with GQ1b (group B), two had IgG anti-GT1a and GQ1b antibodies which cross reacted with each other (group C), and one had a marked increase in IgG anti-GM1b antibodies (group A).

Table 3 Absorption rate of $\operatorname{Ig} G$ anti-GM1b antibody and $\operatorname{Ig} G$ anti-GT1a antibody

\begin{tabular}{llllll}
\hline Absorber & GM1 & GM1b & GT1a & GD1a & GQ1b \\
\hline Patient & Absorption & rate of IgG & anti-GM1b & Ab $(\%)$ & Absorber \\
1 & 6 & 96 & 2 & 92 & 1 \\
2 & 23 & 55 & 0 & 2 & 0 \\
3 & 13 & 82 & 0 & 6 & 0 \\
4 & 0 & 67 & 0 & 0 & 0 \\
Patient & Absorption & rate of IgG & anti-GT1a Ab(\%) & Absorber & \\
5 & 0 & 0 & 75 & 0 & 0 \\
6 & 0 & 0 & 80 & 0 & 0 \\
8 & 0 & 4 & 70 & 39 & 59 \\
9 & 0 & 0 & 81 & 0 & 37 \\
10 & 3 & 14 & 78 & 11 & 98 \\
\hline
\end{tabular}

RELATION OF ANTI-GM1b ANTIBODIES TO BULBAR PALSY

IgG anti-GM1b antibody titres of 500 or more were found in $44(25 \%)$ of the 175 patients with GBS or FS, and IgM antibodies in 21 $(12 \%)$. Fifty three $(30 \%)$ of the patients had bulbar palsy. There was no significant association of bulbar palsy with anti-GM1b IgG antibodies $\left(\chi^{2}\right.$ test $\mathrm{p}=0.80$; Mann-Whitney $U$ test $\mathrm{p}=0.44)$ or the $\operatorname{IgM}\left(\chi^{2}\right.$ test $\mathrm{p}=0.75$; Mann-Whitney $U$ test $\mathrm{p}=0.65$ ).

\section{Discussion}

We often found serum antiganglioside antibodies in patients with GBS or its variants in whom bulbar palsy appeared early in the illness. Measurement of these autoantibodies should be useful to distinguish GBS with early involvement of oropharyngeal functions from such other disorders as botulism and myasthenia gravis. Of the antiganglioside antibodies, IgG anti-GT1a and anti-GM1b seem to be particularly valuable as diagnostic markers for GBS and its variants in which there is early appearance of bulbar palsy, because five (38\%) of 13 patients (patients 2-6) had very high antibody activity to GT1a or GM1b, and low or no activity to other gangliosides. Conversely, measurement of anti-GM1 and anti-GalNAcGD1a antibodies does not seem useful for distinguishing GBS from other diseases when bulbar palsy appears early in the clinical course. All five patients (2-6) with high IgG antibody titre only to GM1b or GT1a had had antecedent diarrhoea, whereas patients $1,8,9$, and 10, with high antibody titres to other gangliosides as well as GM1b and GT1a, often had a history of prior upper respiratory infection. In patients with a history of antecedent diarrhoea in particular, measurement of serum IgG antibodies to gangliosides other than GM1b and GT1a may give false negative results in serological examinations.

GT1a and GM1b are minor human brain gangliosides, ${ }^{14}{ }^{24}$ that also seem to be present on human peripheral nerves. ${ }^{1416}$ The gram negative bacterium Campylobacter jejuni, a leading agent of antecedent infection in GBS, ${ }^{25}$ was isolated from a stool specimen of one patient (3), who had increased IgG antibody titres against GM1b and GT1a. Some strains of $C$ jejuni that had been isolated from patients with GBS have lipopolysaccharides bearing sugars 
that mimic those of GT1 $a^{2627}$ and GM1b. ${ }^{15}$ Molecular mimicry therefore may function in the induction of anti-GT1a and anti-GM1b antibodies which cause neurological deficits such as bulbar palsy in some patients with GBS or its variants, but anti-GT1 $\mathrm{a}^{16}{ }^{17}$ and antiGM1b antibodies are not associated with the presence of bulbar palsy in most patients.

Unlike patients with classic ascending GBS, seven $(54 \%)$ of the 13 patients in this study showed neck and arm dominant muscle weakness, originally described by Ropper in PCB, ${ }^{2}$ whereas the tendon reflex was preserved in the legs of only one patient. Although most of the patients with PCB described by Ropper et $a l^{7}$ made slow recoveries, the bulbar palsy in most of the patients in our study completely disappeared. Limb weakness rather than bulbar palsy remained as a residual symptom in some patients who had early bulbar palsy.

Mizoguchi et $a l^{19}$ and ourselves ${ }^{17}{ }^{21}$ reported the detection of IgG anti-GT1a antibody which does not cross react with GQ1b in PCB. We have now confirmed that anti-GT1a antibody which cross reacts with GQ1b and anti-GM1b antibody is also present in some patients with PCB-like symptoms. In three patients with acute oropharyngeal palsy as described by O'Leary et $a l^{20}$ serum IgG antibodies against GT1a and GQ1b were found during the acute phase of the illness. None of the patients had ophthalmoplegia during the clinical course, even though IgG anti-GQ1b and anti-GT1a antibodies are closely associated with the presence of ophthalmoplegia. ${ }^{28}$ In another patient with GBS, described by Mizoguchi et al, ${ }^{29}$ who had bulbar palsy and generalised muscle weakness but no ophthalmoplegia, the IgG anti-GQ1b and anti-GT1a antibody titres were also raised. We have confirmed that patients who initially had bulbar palsy and later ophthalmoparesis also had raised IgG antibody titres to GQ1b and GT1a as do patients with "typical" FS. We speculate that the involvement of the oculomotor nerves in acute oropharyngeal palsy depends on the severity of the illness and that acute oropharyngeal palsy with increased serum IgG antibody titres to GQ1b and GT1a can be defined as an early clinical stage of FS, $\mathrm{BBE}$, or PCB.

We thank Dr S Abeta (Kyorin University School of Medicine, Tokyo), Dr K Chida (Kohnan Hospital, Miyagi), Dr K Oh Narita Red Cross Hospital, Chiba), Dr T Watanabe (Niigata City Hospital, Niigata), and Dr S Yamashita (Kumamoto City Hospital, Kumamoto) for providing the information on their patients. We also thank Ms Y Tsuchiya (Dokkyo University School of Medicine, Tochigi) for her technical assistance in the testing of antiganglioside antibody. This research was supported in part by grants in aid from the Nakabayashi Trust for ALS Research, and a research grant for neuroimmunological diseases from the Ministry of Health and Welfare of Japan.

1 Hughes TAT, Wiles CM. Neurogenic dysphagia: the role of the neurologist. F Neurol Neurosurg Psychiatry 1998;64:569the ne.
72 .

2 Ropper AH. Unusual clinical variants and signs in Guillain-

Barré syndrome. Arch Neurol 1986;43:1150-2.

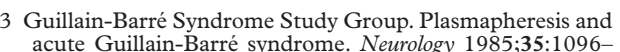
104.

4 French Cooperative Group on Plasma Exchange in Guillain-Barré Syndrome. Efficiency of plasma exchange in Guillain-Barré syndrome: role of replacement fluids. Ann Neurol 1987;22:753-61.

5 van der Meché FGA, Schmitz PIM, the Dutch GuillainBarré Study Group. A randomized trial comparing intravenous immune globulin and plasma exchange in

6 Plasma Exchange/Sandoglobulin Guillain-Barré Syndrome Trial Group. Randomised trial of plasma exchange, intravenous immunoglobulin, and combined treatments in intravenous immunoglobulin, and combined treatm

7 Ropper AH, Wijdicks EFM, Truax BT. Guillain-Barré syndrome. Philadelphia: FA Davis, 1991:18-21, 106-21.

8 Yuki N, Yoshino $\mathrm{H}$, Sato S, et al. Acute axonal polyneuropathy associated with anti-GM1 antibodies following Campylobacter enteritis. Neurology 1990;40:1900-2.

9 Yuki N, Yoshino H, Sato S, et al. Severe acute axonal form of Guillain-Barré syndrome associated with IgG anti-GD1a antibodies. Muscle Nerve 1992;15:899-903.

10 Rees JH, Gregson NA, Hughes RAC. Anti-ganglioside GM1 antibodies in Guillain-Barré syndrome and their relationship to Campylobacter jejuni infection. Ann Neurol 1995;38:809-16.

11 Carpo M, Nobile-Orazio E, Meucci N, et al. Anti-GD1a ganglioside antibodies in peripheral motor syndromes. Ann Neurol 1996;39:539-43.

12 Kusunoki S, Chiba A, Kon K, et al. $\mathrm{N}$-acetylgalactosaminyl GD1a is a target molecule for serum antibody in GuillainBarré syndrome. Ann Neurol 1994;35:570-6.

13 Yuki N, Taki T, Handa S. Antibody to GalNAc-GD1a and GalNAc-GM1b in Guillain-Barré syndrome subsequent to Campylobacter jejuni enteritis. F Neuroimmunol 1996;71: 155-61.

14 Kusunoki S, Iwamori M, Chiba A, et al. GM1b is a new member of antigen for serum antibody in Guillain-Barré syndrome. Neurology 1996;47:237-42.

15 Yuki N, Tagawa Y, Irie F, et al. Close association of GuillainBarré syndrome with antibodies to minor monosialogangliosides GM1b and GM1 a. F Neuroimmunol 1997;74:30-

6 Ilyas AA, Cook SD, Mithen FA, et al. Antibodies to GT1a ganglioside in patients with Guillain-Barré syndrome. $\mathcal{F}$ 88;82:160-7.

17 Koga M, Yuki N, Ariga T, et al. Is IgG anti-GT1a antibody associated with pharyngeal-cervical-brachial weakness or oropharyngeal palsy in Guillain-Barré syndrome? $\mathcal{F} \mathrm{Neu}$ roimmunol 1998;86:74-9.

18 Jacobs BC, van Doorn PA, Schmitz PIM, et al. Campylobacter jejuni infections and anti-GM1 antibodies in Guillain-Barré syndrome. Ann Neurol 1996;40:181-7.

19 Mizoguchi K, Hase A, Obi T, et al. Two species of antiganglioside antibodies in a patient with a pharyngeal-cervicalbrachial variant of Guillain-Barré syndrome. $\mathcal{F}$ Neurol Neurosurg Psychiatry 1994;57:1121-3.

20 O'Leary CP, Veitch J, Durward WF, et al. Acute oropharyngeal palsy is associated with antibodies to GQ1b and GT1a gangliosides. $\mathcal{F}$ Neurol Neurosurg Psychiatry 1996;61:64951 .

21 Kashihara K, Shiro Y, Koga M, et al. IgG anti-GT1a antibodies which do not cross-react with GQ1b ganglioside an a pharyngeal-cervical-brachial variant of Guillain-Barre syndrome. F Neurol Neurosurg Psychiatry 1998:65:799.

22 Asbury AK, Cornblath DR. Assessment of current diagnostic criteria for Guillain-Barré syndrome. Ann Neurol 1990; 27(suppl):S21-4.

23 Yuki N. Acute paresis of extraocular muscles associated with IgG anti-GQ1b antibody. Ann Neurol 1996;39:668-72.

24 Ando S, Yu RK. Isolation and characterization of a novel trisialoganglioside, GT1a, from human brain. $\mathcal{F}$ Biol Chem 1977;252:6247-50.

25 Rees JH, Soudain SE, Gregson NA, et al. Campylobacter jejuni infection and Guillain-Barré syndrome. $N$ Engl $\mathcal{F}$ Med 1995;333:1374-9.

26 Aspinall GO, McDonald AG, Pang H, et al. Lipopolysaccharides of Campylobacter jejuni serotype O:19: structures of rides of Campylobacter jejuni serotype O:19: structures of bacterial isolates from patients with Guillain-Barré synbacterial isolates from patients with
drome. Biochemistry 1994;33:241-9.

27 Yuki N, Handa S, Tai T, et al. Ganglioside-like epitopes of lipopolysaccharides from Campylobacter jejuni (PEN 19) in three isolates from patients with Guillain-Barré syndrome. F Neurol Sci 1995;130:112-6.

28 Chiba A, Kusunoki S, Obata H, et al. Serum anti-GQ1b IgG antibody is associated with ophthalmoplegia in Miller Fisher syndrome and Guillain-Barré syndrome: clinical and immunohistochemical studies. Neurology 1993;43: 1911-7

29 Mizoguchi K, Uchiyama T, Obi T, et al. Anti-GQ1b and anti-GT1a IgG antibodies in a patient with acute demyelinating polyradiculoneuropathy without ophthalmoplegia. $\mathcal{f}$ Neurol Neurosurg Psychiatry 1997;63:410-1. 